\title{
Recurrence after neoadjuvant chemoradiation and surgery for esophageal cancer: Does the pattern of recurrence differ for patients with complete response and those with partial or no response?
}

\author{
Robert A. Meguid, MD, MPH, ${ }^{\mathrm{a}, *}$ Craig M. Hooker, MPH, ${ }^{\mathrm{b}, *}$ Joshua T. Taylor, BS, ${ }^{\mathrm{a}}$ \\ Laurence R. Kleinberg, MD, ${ }^{b}$ Stephen M. Cattaneo II, MD, ${ }^{a}$ Marc S. Sussman, MD, ${ }^{a}$ \\ Stephen C. Yang, MD, ${ }^{\mathrm{a}}$ Richard F. Heitmiller, MD, ${ }^{\mathrm{c}}$ Arlene A. Forastiere, MD, ${ }^{\mathrm{b}}$ and \\ Malcolm V. Brock, MD
}

Objective: We hypothesized that most relapses in patients with esophageal cancer having neoadjuvant chemoradiation therapy would occur outside of the surgical and radiation fields.

Methods: Recurrence patterns, time to recurrence, and median survival were examined in 267 patients who had esophagectomy after neoadjuvant chemoradiation therapy at Johns Hopkins over 19 years.

Results: Of 267 patients, 82 (30.7\%) showed complete response to neoadjuvant therapy, with $108(40.4 \%)$ and 77 $(28.8 \%)$ showing partial response or no response, respectively. Recurrence developed in 84 patients (patients with complete response $18 / 82,21.4 \%$; patients with partial response $39 / 108,36.1 \%$; patients with no response $27 / 77$, $35.1 \% ; P=.055$, respectively). Most patients had recurrences at distant sites $(65 / 84 ; 77.4 \%)$ regardless of pathologic response, and subsequent survival was brief (median 8.37 months). Median disease-free survival was short (10 months) and did not differ based on recurrence site for patients with partial response or no response, but was longer for patients with complete response with distant recurrence, whose median disease-free survival was 27.3 months $(P=.008)$. By multivariate analysis, no other factor except for pathologic response to neoadjuvant therapy was associated with disease recurrence or death. Patients with partial response or no response were 1.97 and 2.23 times more likely to have recurrence than patients with complete response $(P=.024$ and $P=.012$, respectively).

Conclusions: Most esophageal cancer recurrences after neoadjuvant therapy and surgery are distant, and survival time after recurrence is short regardless of pathologic response. Fewer patients achieving complete response had recurrences, and distant recurrences in these patients manifest later than in patients showing partial response and those showing no response. Only pathologic response is significantly associated with disease recurrence, suggesting that tumor biology and chemosensitivity are critical in long-term patient outcome. (J Thorac Cardiovasc Surg 2009;138:1309-17)

Earn CME credits at

http://cme.ctsnetjournals.org
From the Division of Thoracic Surgery, Department of Surgery, ${ }^{\text {a }}$ and Department of Oncology, ${ }^{b}$ Johns Hopkins School of Medicine; and Department of Thoracic Surgery, ${ }^{\mathrm{c}}$ Union Memorial Hospital, Baltimore, Md.

Disclosures: R.A.M. was supported on the Ruth L. Kirschstein National Research Service Award (T32DK007713) while undertaking this study. M.V.B. was supported in part for this work by National Institutes of Health Award (NIH 1R33CA127055-01).

Presented at the annual meeting of Western Thoracic Surgical Association, Kona, Hawaii, June 25, 2008.

* R.A.M. and C.M.H. contributed equally to this article.

Received for publication July 7, 2008; revisions received June 26, 2009; accepted for publication July 29, 2009.

Address for reprints: Dr Malcolm V. Brock, Division of Thoracic Surgery, Department of Surgery, 600 North Wolfe St, Blalock 240, The Johns Hopkins Hospital, Baltimore, MD 21287 (E-mail: mbrock1@jhmi.edu).

$0022-5223 / \$ 36.00$

Copyright (c) 2009 by The American Association for Thoracic Surgery

doi:10.1016/j.jtcvs.2009.07.069
Most surgical candidates with esophageal cancer present with regional nodal metastases, or stage III disease. ${ }^{1}$ Historically, the primary curative treatment was a total or partial esophagectomy and lymph node dissection, with resultant 5-year survival rates ranging between $15 \%$ and $20 \% .{ }^{1}$ After encouraging preliminary data in the late $1980 \mathrm{~s}$, and our own investigations, neoadjuvant chemoradiotherapy in combination with surgery became the mainstay of treatment at our institution over the last 19 years. This trimodality approach is now widely accepted clinical practice. ${ }^{2,3}$

Despite this widespread use, especially in North America, short- and long-term patient outcome data after neoadjuvant therapy plus surgery have been conflicting. ${ }^{4,5}$ Importantly, however, the $25 \%$ to $30 \%$ of patients with a pathologic complete response (CR) after this therapy have significantly improved 5-year survival rates compared with other treatment modalities for advanced esophageal cancer. ${ }^{4-7}$ However, it is also clear that about $30 \%$ of patients with a pathologic CR still have recurrences and die from metastatic esophageal cancer. ${ }^{8}$ In these patients with a pathologic 


\section{Abbreviations and Acronyms \\ AJCC $=$ American Joint Committee on Cancer \\ $\mathrm{CR}=$ complete response \\ $\mathrm{NR}=$ nonresponse \\ $\mathrm{PR}=$ partial response}

$\mathrm{CR}$, in particular, little is known about the impact of neoadjuvant chemoradiation on the timing and patterns of recurrent esophageal cancer or on the overall survival of these patients with recurrent disease.

We hypothesized that chemoradiation plus surgery would lead to improved local tumor control compared with surgery alone and that most relapses would occur outside the surgical and radiation fields. We also hypothesized that recurrence would predominate in patients with chemoresistant tumors that were not impacted by neoadjuvant chemoradiation and surgery, and thus would occur relatively quickly. We, therefore, examined if recurrence patterns, time to recurrence, and overall median survival differed with pathologic response to neoadjuvant chemoradiotherapy.

\section{METHODS \\ Data Source}

This is a retrospective analysis using patient data collected from the Johns Hopkins Hospital Multidisciplinary Esophageal Cancer Database, which contains demographic and clinical data and outcome of patients with esophageal cancer evaluated at Johns Hopkins Hospital between January 1, 1989, and December 31, 2007. Data in the database were obtained from patient hospital charts, as well as electronic and paper files. Since 2000, periodic data audits have been performed by archival and online chart reviews for quality assurance. These reviews have consistently verified over $90 \%$ accuracy of the database with source materials.

\section{Patient Outcome Data}

Follow-up data and sites of tumor recurrence were determined by a systematic follow-up of any patients with incomplete data attained by letters, E-mails, faxes, and phone calls to their referring physicians. In almost all cases, recurrence was documented radiographically, but, in a few cases, there was also pathologic assessment. All sites of first recurrence were recorded. These data were obtained from clinic charts with confirmation by reviewing associated imaging and pathology reports. This study was approved by the Johns Hopkins Institutional Review Board, which exempted the need for patient consent, and abides by Health Insurance Portability and Accountability Act compliance standards.

\section{Patient Population}

Patient selection criteria included a biopsy-proven esophageal carcinoma and a documented assessment by a thoracic surgeon, a medical oncologist, a gastroenterologist, and a radiation oncologist at the Johns Hopkins Hospital. We identified 267 patients who had pretreatment clinical staging, neoadjuvant chemotherapy and/or radiotherapy, and an esophagectomy with curative intent.

Race was self-reported as Caucasian, African-American, or other. For ease of analysis, patients of other race were documented as minorities and combined with the African-American patients. Clinical staging was determined at the time of initial evaluation and was available on all but 2 patients who both showed complete response. All 267 patients had a recorded pathologic stage derived from the pathology record. Smoking history was available on all but 5 patients. Patients were identified as ever or never smokers based on self-reporting. Ever smokers were defined as those who had smoked 100 tobacco cigarettes or more during their lifetime, and never smokers were those who had smoked fewer than 100 cigarettes. Pack-year index was determined by multiplying the average number of cigarette packs smoked per day by the number of years smoked. The American Society of Anesthesiologists 5 -grade classification system was used as an index of preoperative comorbidity. ${ }^{9}$ Esophagectomies were performed as partial esophagectomies using the transhiatal $(81 \%)$, the 3 -incision $(15 \%)$, the Ivor Lewis $(2 \%)$, or the thoracoabdominal approach ( $2 \%$; Table 1). During the length of the study, these 4 approaches were all used with concomitant mediastinal lymphadenectomy. The median number of lymph nodes harvested was 15 .

\section{Neoadjuvant Chemoradiation and Pathologic Response}

To compare and control for multimodal preoperative and postoperative therapy, we examined neoadjuvant chemo- and radiotherapy types and doses as well as adjuvant chemotherapy. Neoadjuvant chemotherapy agents were almost always given as platinum-based doublets and are stratified as either with or without fluorouracil. Neoadjuvant radiotherapy dose was recorded as either $<4400 \mathrm{cGy}$ or $\geq 4400 \mathrm{cGy}$. Postoperative adjuvant chemotherapy was recorded as either administered or not.

Pathologic response to neoadjuvant chemoradiation was based on our previously described classification system and determined at the time of pathologic examination of the surgical specimen. ${ }^{10}$ Briefly, patients were identified as having a pathologic CR if no microscopic evidence of tumor was found upon examination of both the resected esophageal specimen and nodal tissues. Partial response (PR) described patients with persistence of microscopic esophageal carcinoma in the resected surgical specimen but their overall American Joint Committee on Cancer (AJCC) ${ }^{11}$ stage was lower as compared with preoperative clinical staging. Nonresponse (NR) described patients with either no change in AJCC stage between clinical and pathologic staging, or progression of disease despite neoadjuvant therapy. Of note, at our institution, we treat patients with stage IVA disease (local lymph node involvement) with multimodality therapy. Preoperative clinical staging was performed by compiling results from computed tomography scans, esophageal endoscopic ultrasound, barium esophagrams, positron emission tomography scans ( $65 \%$ of patients), and in some patients, exploratory laparoscopy. ${ }^{10}$

\section{Recurrences}

Time between diagnosis and surgery, length of stay after surgery, recurrence-free survival (time from surgery to disease recurrence), and overall survival were calculated and reported. All patients in the data set were included in consideration of disease recurrence (or disease progression for the patients with no response to neoadjuvant chemoradiation) except 6 patients who were found at time of surgery to have metastatic stage IVA (2 patients with celiac lymph nodes) or IVB disease ( 2 patients with isolated liver metastases, 1 patient with an omental metastasis, and 1 patient with supraclavicular disease). Note that these 6 patients still were aggressively resected and local therapy applied to their metastatic site, and so they are included in the larger cohort of 267 patients. All sites of tumor recurrence were recorded and categorized as the abdomen (excluding the liver), the liver, the brain, bone, the chest (excluding the esophagus), the esophagus, and other.

\section{Statistical Analysis}

Comparison of continuous, categorical, and dichotomous variables was performed using the Student $t$ test and chi-square test for homogeneity. Time to recurrence-free survival and all-cause mortality was modeled using the Kaplan-Meier method, and the association of factors with time to recurrence and death was analyzed using the Cox proportional hazards model. The model was adjusted by risk factors for recurrence and mortality that 
TABLE 1. Characteristics of the study population by pathologic response $(n=267)$

\begin{tabular}{|c|c|c|c|c|c|c|c|}
\hline & \multicolumn{2}{|c|}{ Complete response, $82(30.7 \%)$} & \multicolumn{2}{|c|}{ Partial response, $108(40.4 \%)$} & \multicolumn{2}{|c|}{ No response, $77(28.8 \%)$} & \multirow[b]{2}{*}{$P$ value } \\
\hline & $\mathbf{n}$ & $\%$ & $\mathbf{n}$ & $\%$ & $\mathbf{n}$ & $\%$ & \\
\hline Age at diagnosis (y), median and IQR & 61 & $51-67 \ddagger$ & 61 & $51-67 \ddagger$ & 60 & $52-67 \ddagger$ & .773 \\
\hline Sex & & & & & & & .002 \\
\hline Male & 66 & 80.5 & 104 & 96.3 & 69 & 89.6 & \\
\hline Female & 16 & 19.5 & 4 & 3.7 & 8 & 10.4 & \\
\hline Race & & & & & & & .404 \\
\hline White & 76 & 92.6 & 102 & 94.4 & 68 & 88.3 & \\
\hline Black & 3 & 3.7 & 4 & 3.8 & 7 & 9.1 & \\
\hline Other & 3 & 3.7 & 2 & 1.8 & 2 & 2.6 & \\
\hline Smoked cigarettes* & & & & & & & .952 \\
\hline Never & 15 & 19.0 & 22 & 2.4 & 14 & 18.7 & \\
\hline Ever & 64 & 81.0 & 86 & 79.6 & 61 & 81.3 & \\
\hline Pack-years smoked (y), ${ }^{*}$ median and IQR & 32.9 & $23.7-53.6 \ddagger$ & 35 & $15-50 \ddagger$ & 40 & $25-60 \ddagger$ & .284 \\
\hline ASA classification, median & 3 & & 3 & & 3 & & .565 \\
\hline Histology & & & & & & & $<.001$ \\
\hline Adenocarcinoma & 54 & 65.9 & 96 & 88.9 & 58 & 75.3 & \\
\hline Squamous cell & 24 & 29.3 & 12 & 11.1 & 19 & 24.7 & \\
\hline Carcinoma, NOS & 4 & 4.8 & 0 & .0 & 0 & 0.0 & \\
\hline Clinical stage $\dagger$ & & & & & & & $<.001$ \\
\hline IIA & 29 & 36.3 & 11 & 1.2 & 33 & 42.9 & \\
\hline IIB & 9 & 11.2 & 15 & 13.9 & 15 & 19.4 & \\
\hline III & 35 & 43.8 & 61 & 56.5 & 23 & 29.9 & \\
\hline IVA & 7 & 8.7 & 21 & 19.4 & 6 & 7.8 & \\
\hline Pathologic stage & & & & & & & $<.001$ \\
\hline No evidence of disease & 82 & 100 & 0 & .0 & 0 & 0.0 & \\
\hline I & 0 & 0.0 & 30 & 27.8 & 0 & 0.0 & \\
\hline IIA & 0 & 0.0 & 60 & 55.6 & 27 & 35.0 & \\
\hline IIB & 0 & 0.0 & 13 & 12.0 & 9 & 11.7 & \\
\hline III & 0 & 0.0 & 5 & 4.6 & 35 & 45.5 & \\
\hline IVA & 0 & 0.0 & 0 & .0 & 2 & 2.6 & \\
\hline IVB & 0 & 0.0 & 0 & .0 & 4 & 5.2 & \\
\hline Neoadjuvant chemotherapy & & & & & & & .149 \\
\hline 5-FU and platinum only & 62 & 75.6 & 70 & 64.8 & 46 & 59.7 & \\
\hline Non-5-FU/platinum & 8 & 9.8 & 21 & 19.5 & 19 & 24.7 & \\
\hline Course unknown & 12 & 14.6 & 17 & 15.7 & 12 & 15.6 & \\
\hline Neoadjuvant radiotherapy dose & & & & & & & .137 \\
\hline$<4,400$ cGy & 3 & 3.7 & 6 & 5.5 & 6 & 7.8 & \\
\hline$\geq 4,400 \mathrm{cGy}$ & 54 & 65.9 & 58 & 53.7 & 39 & 50.6 & \\
\hline Dose unknown & 25 & 30.4 & 43 & 39.8 & 28 & 36.4 & \\
\hline No radiotherapy & 0 & 0.0 & 1 & 1.0 & 4 & 5.2 & \\
\hline Adjuvant chemotherapy & & & & & & & .230 \\
\hline Received & 19 & 23.2 & 26 & 24.1 & 11 & 14.3 & \\
\hline Not received & 63 & 76.8 & 82 & 75.9 & 66 & 85.7 & \\
\hline Surgical procedure & & & & & & & .522 \\
\hline Transhiatial & 65 & 79.3 & 88 & 81.5 & 62 & 80.5 & \\
\hline 3-Incision & 14 & 17.1 & 17 & 15.7 & 10 & 13.0 & \\
\hline Ivor Lewis & 3 & 3.6 & 1 & 0.9 & 2 & 2.6 & \\
\hline Thoracoabdominal & 0 & 0.0 & 2 & 1.9 & 3 & 3.9 & \\
\hline $\begin{array}{l}\text { Interval between diagnosis and } \\
\text { surgery, (days) median and IQR }\end{array}$ & 91 & $82-108$ & 94 & $82-110$ & 90 & $78-110$ & .730 \\
\hline Postoperative stay, (days) median and IQR & 9 & 8-14 & 9 & $8-10$ & 8 & $7-11$ & .565 \\
\hline Overall survival, (mos) median and IQR & 79.3 & $30.1-\mathrm{NR}$ & 30.6 & $14.8-94.9$ & 18.6 & $11.0-40.8$ & .001 \\
\hline $\begin{array}{l}\text { Overall survival after recurrence, } \\
(\mathrm{mos}) \text { median and IQR }\end{array}$ & 10.8 & $2.6-21.1$ & 9.8 & $3.1-16.6$ & 7.2 & $4.7-14.4$ & .707 \\
\hline
\end{tabular}

$\overline{A S A}$, American Society of Anesthesiologists; $F-F U$, fluorouracil; $I Q R$, interquartile range; $N O S$, not otherwise specified; $N R$, not reached. $*$ Available on 262 patients. $\dagger$ Available on 265 patients. †़Interquartile range. 
TABLE 2. First tumor recurrence site according to pathologic response status to neoadjuvant therapy $(\mathbf{n}=84)$

\begin{tabular}{|c|c|c|c|c|c|c|c|}
\hline & \multicolumn{6}{|c|}{ Proportion of patients with recurrence from total cohort } & \multirow[b]{3}{*}{$P$ value } \\
\hline & \multicolumn{2}{|c|}{ Complete response $(n=82)$} & \multicolumn{2}{|c|}{ Partial response $(n=108)$} & \multicolumn{2}{|c|}{ No response $(n=77)$} & \\
\hline & $\mathbf{n}$ & $\%$ & $\mathbf{n}$ & $\%$ & $\mathbf{n}$ & $\%$ & \\
\hline Overall recurrence & 18 & 22.0 & 39 & 36.1 & 27 & 35.1 & .055 \\
\hline \multicolumn{8}{|l|}{ Tumor site } \\
\hline Esophagus and mediastinum & 5 & 27.2 & 8 & 20.5 & 6 & 22.2 & \\
\hline Lung and pleura & 4 & 22.2 & 7 & 17.9 & 4 & 14.8 & \\
\hline Abdomen & 5 & 27.7 & 9 & 23.0 & 4 & 14.8 & \\
\hline Liver & 1 & 5.5 & 6 & 15.3 & 6 & 22.2 & \\
\hline Brain & 1 & 5.5 & 2 & 5.1 & 2 & 7.4 & \\
\hline Bone & 2 & 11.1 & 7 & 17.9 & 5 & 18.5 & \\
\hline
\end{tabular}

included patient sex, histology, and clinical stage. Crude hazard ratios and corresponding $95 \%$ confidence intervals are reported. All hazard ratios are presented as independent variables and then further adjusted for specified variables and are presented as adjusted hazard ratios.

Overall survival was defined as time from date of surgical therapy to death or last follow-up. Disease-free survival, defined as time from date of surgical therapy to first recurrence or last follow-up, is used interchangeably with "recurrence-free" survival in the article.

Differences between Kaplan-Meier survival curves were estimated using the log-rank test. Statistical analysis was performed using the software package STATA 10.0 (StataCorp, College Station, Tex).

\section{RESULTS}

Table 1 shows the clinicopathologic characteristics of all 267 patients. Median age was 60 years (interquartile range, 51-67) with $239(89.5 \%)$ being men. The racial distribution included $246(92.2 \%)$ Caucasians, 14 (5.2\%) African-Americans, and 7 (2.6\%) of other race. Most patients, $211(80.5 \%)$, were ever smokers (87 current smokers; 124 former smokers) with a median pack-years smoked of 35. Adenocarcinoma was the reported histology for $208(77.9 \%)$ patients, squamous cell histology for 55 $(20.6 \%)$ patients, and $4(1.5 \%)$ patients with carcinoma, not otherwise specified. Median time between diagnosis and surgery was not different between response groups, nor was there a difference in median hospital length of stay. In patients with known chemotherapy regimens and radiation doses, the majority received fluorouracil and platinum neoadjuvant chemotherapy (178/226, 78.8\%), along with at least $4400 \mathrm{cGy}(151 / 166,91 \%)$ of neoadjuvant radiation. Only 56/267 (21.0\%) of patients received adjuvant chemotherapy.
Of 267 patients, $82(30.7 \%)$ had CR to neoadjuvant therapy, 108 (40.4\%) patients had PR, and 77 (28.8\%) had NR. On univariate analysis, PR, CR, and NR cohorts differed significantly by sex, tumor histology, and initial clinical stage (Table 1). Esophageal malignancy recurred in a total of 84 patients $(31.5 \%)$. There was a strong trend toward a statistically significant difference in frequency of esophageal recurrence between response groups (CR: 18/82, 22.\%; PR: 39/ 108, 36.1\%; and NR: 27/77, 35.5\%; $P=.055$; Table 2). The distributions of patterns of first tumor recurrence for the individual organ sites between the 3 different pathologic response groups are also presented in Table 2. Although there seems to be proportionately fewer bone/liver metastases for the patients in the CR group, there is no significant difference in site of recurrences between response groups. When recurrence sites were grouped as either within the radiation field (including esophagus and mediastinum) versus outside the field (all other sites of recurrence), again there was no significant difference in patterns of recurrence according to the pathologic response group (Table 3).

Median disease-free survival differed significantly when stratified by response group and whether or not the first site of recurrence was locoregional or distant (Table 4). All recurrences occurred within a median time of 10 months postoperatively except for distant recurrences in patients who had a CR to neoadjuvant chemoradiation. In these patients, the median disease-free survival was 27.3 months $(P=.008)$. Thus, these data suggest that neoadjuvant chemoradiation delayed recurrence significantly and as well fewer patients failed $(P=.055)$. Figure 1 showing freedom

TABLE 3. Proportion of first recurrences within and outside the radiation field according to pathologic response $(n=84)$

\begin{tabular}{|c|c|c|c|c|c|c|c|}
\hline & \multicolumn{6}{|c|}{ Proportion recurred } & \multirow[b]{3}{*}{$P$ value } \\
\hline & \multicolumn{2}{|c|}{ Complete response $(n=18)$} & \multicolumn{2}{|c|}{ Partial response $(n=39)$} & \multicolumn{2}{|c|}{ No response $(n=27)$} & \\
\hline & $\mathbf{n}$ & $\%$ & $\mathbf{n}$ & $\%$ & $\mathbf{n}$ & $\%$ & \\
\hline Radiation field & & & & & & & .829 \\
\hline Within radiation field & 5 & 27.8 & 8 & 20.5 & 6 & 22.2 & \\
\hline Outside radiation field & 13 & 72.2 & 31 & 79.4 & 21 & 77.8 & \\
\hline
\end{tabular}


TABLE 4. Median disease-free survival (in months) of patients with recurrence $(n=84)$

\begin{tabular}{|c|c|c|c|c|c|c|c|}
\hline & \multicolumn{2}{|c|}{ Complete response } & \multicolumn{2}{|c|}{ Partial response } & \multicolumn{2}{|c|}{ No response } & \multirow[b]{2}{*}{$P$ value } \\
\hline & Median & IQR & Median & IQR & Median & IQR & \\
\hline Within radiation field & 5.8 & $4.7-9.3$ & 7.1 & $3.5-18$ & 8.5 & $7.5-62.3$ & .604 \\
\hline Outside radiation field & 27.3 & $12.3-31.1$ & 5.7 & $3.9-15.8$ & 9.1 & $6.9-14.5$ & .008 \\
\hline
\end{tabular}

$I Q R$, Interquartile range.

from recurrence again underscores the fact that patients with CR have a significantly longer disease-free interval than patients with PR and NR $(P=.01)$.

This impact of chemoradiation in preventing recurrence was of sufficient magnitude also to impact survival. The median overall survival was greatest for the CR cohort (79.3 months), which was significantly longer than that of the PR (30.6 months) and NR cohorts (18.6 months), respectively $(P<.001)$. Kaplan-Meier overall survival estimates of all 3 pathologic response groups are depicted in Figure 2, showing a statistically significant difference in overall survival (all-cause mortality) at 5 years. Interestingly, early mortality $(<10$ months) did not differ by pathologic response group, with only patients surviving longer than 1 year showing significant differences in mortality by pathologic response to neoadjuvant therapy (Figure 2). The overall 5-year survival for patients with CR and PR who did not recur is statistically longer than for those patients whose cancer recurred (Figures 3 and 4). Patients in the NR group who did not progress with further disease had survival outcomes as poor as those who showed clinical evidence of disease progression (Figure 5). This is probably because if tumors are aggressive enough to be recalcitrant to neoadjuvant chemoradiation, little additional tumor burden is necessary to cause eventual death.

After adjusting for sex, histology, and clinical stage in a multivariate model, no other demographic or clinicopathologic factor except for pathologic response to neoadjuvant

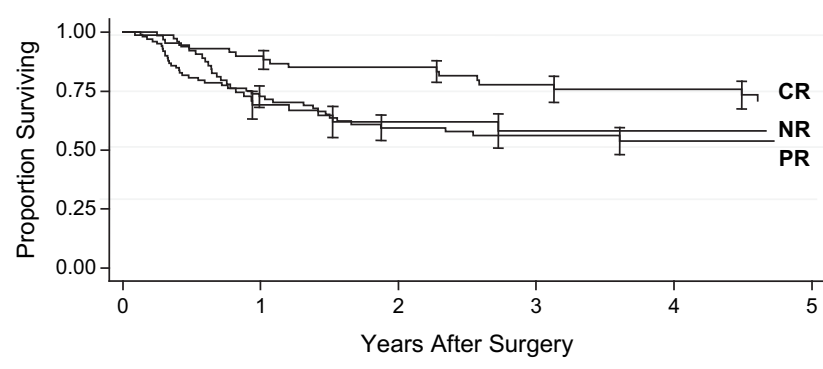

No. at Risk:

\begin{tabular}{|l|c|l|l|l|l|}
\hline CR & 82 & 57 & 49 & 38 & 32 \\
PR & 108 & 61 & 38 & 27 & 23 \\
\hline NR & 71 & 37 & 20 & 15 & 12 \\
\hline
\end{tabular}

FIGURE 1. Kaplan-Meier estimates of recurrence-free survival of patients with esophageal cancer $(n=261)$ at the Johns Hopkins Hospital, according to pathologic response at time of surgical resection. $C R$, Complete response; $N R$, nonresponse; $P R$, partial response. therapy at time of surgical resection was associated with disease-free survival. Patients in the PR and NR groups were 1.97 and 2.23 times more likely to have disease recurrence $(P=.024$ and $P=.012$, respectively; Table 5). Once disease recurred, all deaths occurred within a year regardless of initial response groups and all deaths were cancer-related (data not shown). Finally, the median overall survival of patients with recurrence versus patients without recurrence was 21.2 months versus 65.2 months, respectively $(P<.01)$.

\section{DISCUSSION}

We report on our institution's experience with esophageal cancer, examining survival outcomes and patterns of disease recurrence after neoadjuvant chemoradiotherapy. For all 3 response groups, most recurrences were distant, suggesting the important role of intensified local therapies to eradicate local disease and prevent locoregional recurrence. By multivariate analysis, only pathologic response to neoadjuvant chemotherapy is a significant factor in disease recurrence. The implication of this finding is that, given eminent resectability of the primary tumor, the esophageal tumor biology with its intrinsic sensitivities to the correct chemoradiation

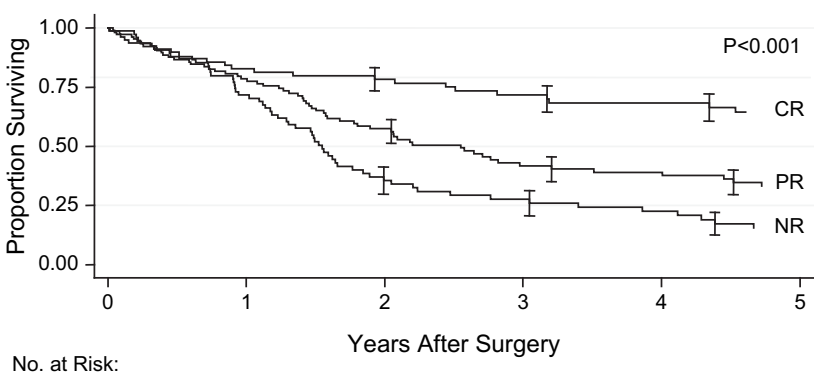

\begin{tabular}{|l|c|c|c|c|c|}
\hline CR & 82 & 59 & 51 & 43 & 36 \\
\hline PR & 108 & 77 & 51 & 33 & 29 \\
\hline NR & 77 & 53 & 24 & 17 & 14 \\
\hline
\end{tabular}

FIGURE 2. Kaplan-Meier estimates of overall survival of patients with esophageal cancer $(n=267)$ at the Johns Hopkins Hospital, according to pathologic response at time of surgical resection. The Kaplan-Meier estimates for overall survival indicate that as the extent of pathologic response to neoadjuvant therapy increases, there is a significant increase in the overall survival of the patient. It is interesting to note, however, that in our observed cohort, the degree of pathologic response to neoadjuvant therapy seemed not to affect patients with early mortality ( $<10$ months) but rather those who survived more than 1 year. $C R$, Complete response; $N R$, nonresponse; $P R$, partial response. 


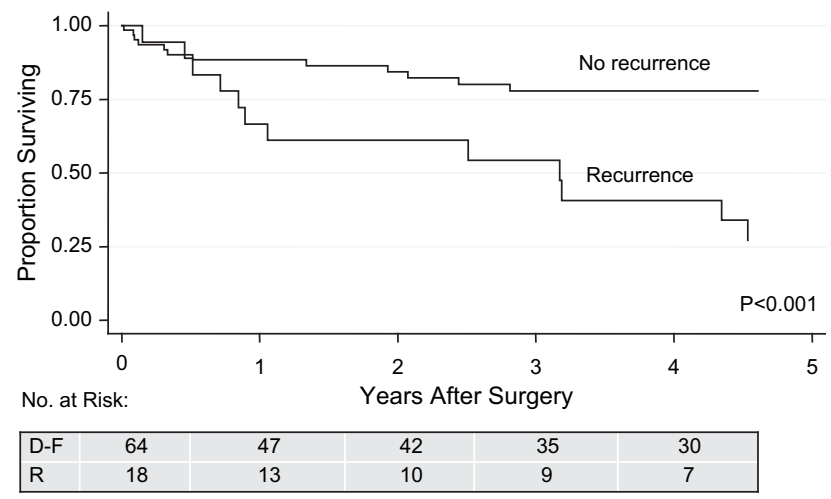

FIGURE 3. Kaplan-Meier estimates of overall survival of patients with esophageal cancer who had a complete pathologic response to neoadjuvant therapy $(n=82)$ at the Johns Hopkins Hospital, according to whether or not there was cancer recurrence following surgical resection. $D-F$, Disease-free; $R$, recurrence.

regimens is the most critical factor in determining patient outcome. Patients showing complete response, even those who eventually have recurrence with distant disease, experienced the longest median recurrence-free survival time, and moreover, patients with a complete response to neoadjuvant therapy had significantly improved overall survival compared with patients with partial response and those with no response. The frequency of recurrence tended to be greater for those showing no response and lowest for patients showing complete response.

In patients with $\mathrm{CR}$, tumors exhibited such a high degree of chemoradiation sensitivity that not only the primary tumor but also distant micrometastases were controlled by the neoadjuvant therapy. It is possible that in patients in the PR group with variable tumor sensitivity, the persistence of distant micrometastases caused distant tumor recurrence.

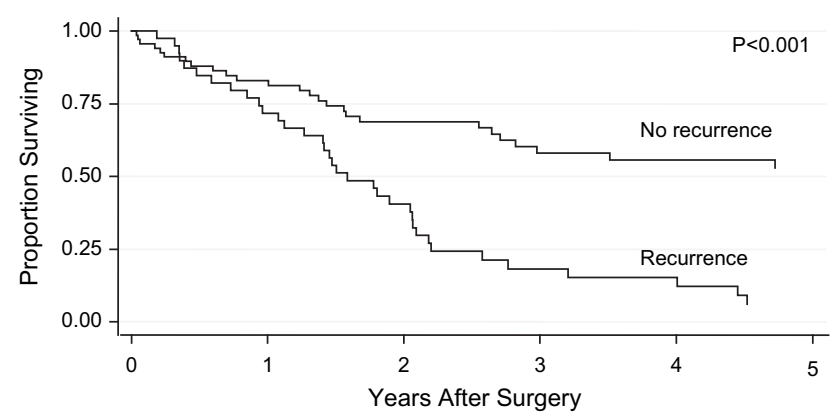

No. at Risk:

\begin{tabular}{|l|c|c|c|c|c|}
\hline $\mathrm{D}-\mathrm{F}$ & 69 & 49 & 36 & 27 & 24 \\
\hline $\mathrm{R}$ & 39 & 29 & 16 & 7 & 6 \\
\hline
\end{tabular}

FIGURE 4. Kaplan-Meier estimates of overall survival of patients with esophageal cancer who had a partial pathologic response to neoadjuvant therapy $(\mathrm{n}=108)$ at the Johns Hopkins Hospital, according to whether or not there was cancer recurrence following surgical resection. $D-F$, Disease-free; $R$, recurrence.

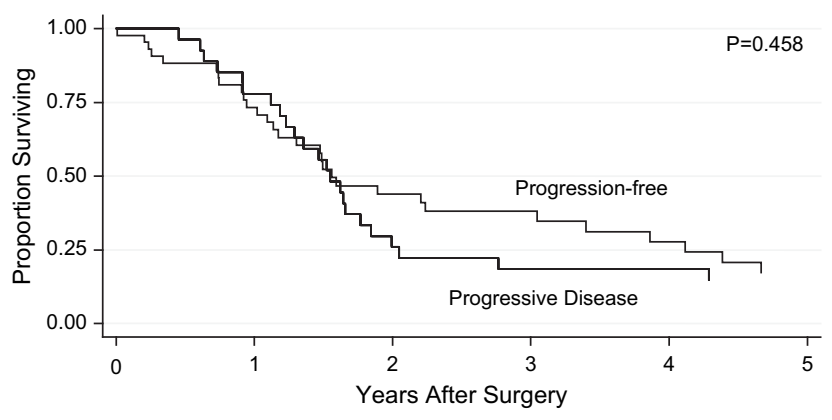

No. at Risk:

\begin{tabular}{|c|c|c|c|c|c|}
\hline D-F & 44 & 30 & 16 & 12 & 9 \\
\hline $\mathrm{R}$ & 27 & 22 & 8 & 6 & 6 \\
\hline
\end{tabular}

FIGURE 5. Kaplan-Meier estimates of overall survival of patients with esophageal cancer who had no pathologic response to neoadjuvant therapy ( $\mathrm{n}=71$ ) at the Johns Hopkins Hospital, according to whether or not there was cancer recurrence following surgical resection. $D-F$, Disease-free; $R$, recurrence.

In an aggressive cancer, such as esophageal cancer, any remaining distant metastasis after neoadjuvant therapy ensured that patients with PR had recurrences outside of the treated field with the same virulence as those patients with NR not affected by neoadjuvant therapy (Table 4 ).

These findings reinforce just how critical a CR can be to a patient with esophageal cancer having neoadjuvant therapy and surgery. The 5 patients who had CR and whose recurrence was local received no discernable benefit from chemoradiation, as the median disease-free interval of these 5 patients was not different from that of the patients who had a PR or NR. This is in direct contrast to the 13 patients who had CR and who recurred distantly after neoadjuvant therapy and surgery, as these patients had late relapse, with a median recurrence time of over 2 years. Presumably, the micrometastatic disease present in these 13 patients with CR had a molecular profile with some degree of chemosensitivity to the preoperative chemotherapeutic regimen given, which presumably delayed the eventual reemergence of distant macrometastases.

The demographic and histopathologic composition of our cohort is representative of other large medical centers with high-volume esophageal practices in the United States over this period. ${ }^{6,7,12}$ Our percentages of patients with esophageal cancer who had a CR, a PR, and NR and who received neoadjuvant therapy is also very similar to that of other groups ${ }^{6,7}$ despite small differences in definitions used for codification of groups. The similarity of our results to others validates the universal nature of our findings.

Although our data showed that locoregional recurrence rates did not differ according to response groups (Table 3), we do not think that these data can be extrapolated to support the assertion that surgery alone may be effective at controlling local disease. Our $23 \%$ local recurrence rate for patients having neoadjuvant chemoradiation closely resembles the 
TABLE 5. Crude and adjusted hazard ratios for the association between pathologic response and recurrence

\begin{tabular}{|c|c|c|c|c|c|c|}
\hline & Crude hazard ratio & $95 \% \mathrm{CI}$ & $P$ value & Adjusted hazard ratio* & $95 \% \mathrm{CI}$ & $P$ value \\
\hline \multicolumn{7}{|l|}{ Clinical response } \\
\hline Complete response & 1.00 & Referent & & 1.00 & Referent & \\
\hline Partial response & 2.13 & $1.22-3.74$ & 0.008 & 1.97 & $1.10-3.57$ & .024 \\
\hline No response & 2.26 & $1.24-4.13$ & 0.008 & 2.23 & $1.20-4.17$ & .012 \\
\hline Age at diagnosis (y) & 0.98 & $0.96-1.00$ & 0.164 & & & \\
\hline \multicolumn{7}{|l|}{ Sex } \\
\hline Male & 1.00 & Referent & & 1.00 & Referent & \\
\hline Female & 0.74 & $0.34-1.60$ & 0.440 & 0.91 & $0.38-2.21$ & .840 \\
\hline \multicolumn{7}{|l|}{ Race } \\
\hline White & 1.00 & Referent & & & & \\
\hline Black & 1.58 & $0.58-4.34$ & 0.369 & & & \\
\hline \multicolumn{7}{|l|}{ Histology } \\
\hline Adenocarcinoma & 1.00 & Referent & & 1.00 & Referent & \\
\hline Squamous cell & 0.85 & $0.45-1.43$ & 0.458 & 0.95 & $0.50-1.83$ & .887 \\
\hline \multicolumn{7}{|l|}{ ASA classification } \\
\hline 2 & 1.00 & Referent & & & & \\
\hline 3 & 0.97 & $0.50-1.88$ & 0.925 & & & \\
\hline $4-5$ & 0.8 & $0.22-2.91$ & 0.734 & & & \\
\hline \multicolumn{7}{|l|}{ Clinical stage } \\
\hline IIA & 1.00 & Referent & & 1.00 & Referent & \\
\hline IIB & 1.42 & $0.74-2.72$ & 0.290 & 1.21 & $0.63-2.35$ & .565 \\
\hline III & 1.21 & $0.72-2.04$ & 0.469 & 1.19 & $0.68-2.07$ & .537 \\
\hline IVA & 1.18 & $0.53-2.65$ & 0.681 & 1.13 & $0.47-2.68$ & .787 \\
\hline \multicolumn{7}{|c|}{ Neoadjuvant chemotherapy } \\
\hline 5-FU-based & 1.00 & Referent & & & & \\
\hline Non-5-FU-based & 0.90 & $0.53-1.51$ & 0.684 & & & \\
\hline \multicolumn{7}{|c|}{ Neoadjuvant radiotherapy } \\
\hline$\geq 4400 \mathrm{cGy}$ & 1.00 & Referent & & & & \\
\hline$<4400$ cGy & 0.47 & $0.15-1.52$ & 0.209 & & & \\
\hline Dose unknown & 0.85 & $0.53-1.36$ & 0.497 & & & \\
\hline \multicolumn{7}{|l|}{ Smoked cigarettes } \\
\hline Never & 1.00 & Referent & & & & \\
\hline Ever & 1.09 & $0.63-1.88$ & 0.755 & & & \\
\hline Pack-years smoked & 0.99 & $0.98-1.01$ & 0.250 & & & \\
\hline
\end{tabular}

ASA, American Society of Anesthesiologists; $C I$, confidence interval; 5-FU, fluorouracil. *Adjusted for sex, histology, and clinical stage.

$19 \%$ local failure rate of the neoadjuvant chemoradiation arm of the randomized clinical trial of Urba and colleagues ${ }^{13}$ conducted at the University of Michigan. Moreover, in both the Michigan trial and the large, randomized US G-I Intergroup trial, the locoregional disease recurrence rates for the surgery alone control arms were $42 \%$ and $58 \%$, respectively. ${ }^{14,15}$ Importantly, our results therefore further corroborate the point that neither surgery alone nor chemoradiation alone is as effective as trimodality therapy in achieving local disease control.

In multivariable regression analysis examining the likelihood of tumor recurrence, patients with a PR or NR to neoadjuvant therapy were 1.97 and 2.23 times more likely to develop tumor recurrence compared with patients with a CR. These data are consistent with varying degrees of tumor resistance to cytotoxic agents and radiotherapy, and correlate with shortened survival and death. Thus pathologic response status was a reasonable surrogate for risk of recurrence and may be useful along with molecular characteristics in the future for selecting patients for additional or alternative forms of therapy following surgery.

Limitations of this study include its retrospective nature, the confines of a single institutional study, and limitations of the database. Hence, specific data points such as cancer-specific death were not able to be obtained on all patients. This limits the ability to determine with certainty that death during follow-up is entirely due to recurrence of tumor. The relatively small size of this study does not allow for rigorous stratification, such as examining differences in outcomes between chemotherapeutic agents and different doses of radiotherapy. In larger databases, however, such as the Nationwide Inpatient Sample and the Surveillance, Epidemiology, and End Results databases, these variables are either characteristically missing or erroneous. Ideally, to study outcomes such as 
those we report, a multi-institutional comprehensive patient-oriented database would be needed. Because this study spans 19 years and multiple examining pathologists, we reported pathologic findings based on a simple standard of CR, PR, and NR rather than the more detailed descriptor of percentage of viable cells in the specimen. A final limitation of the study is that because of its retrospective nature, the time to recurrence (and location of recurrences) could have been impacted by the fastidiousness of monitoring.

The findings of this study, and others, ${ }^{16}$ suggest that not all esophageal carcinomas are equal and that rather than more aggressive chemotherapy regimens, or more intensive radiation dosing schedules, efforts must be made to target different tumors with chemotherapeutic agents to which they are sensitive. This new era of "personalizing" or customizing chemotherapeutic and radiation regimens to specific molecular targets in the primary tumor is now upon us, and because of the success of the neoadjuvant therapy approach in esophageal cancer, this is an appropriate cancer model to test these innovations. Importantly, this study emphasizes the critical role of neoadjuvant chemotherapy in "eliminating" distant disease outside of the local surgery and radiation fields and offers yet more evidence that ultimately the control of distant disease is critical in preventing esophageal cancer recurrence. Patients who experience a CR to neoadjuvant therapy have the best outcomes in terms of lowest incidence of recurrence, longest disease-free survival, and longest overall survival.

The authors would like to acknowledge the Hosler Family, the Thorn Family, the family of Mr Robert Carollo, and the family of Mrs Catherine Remmert whose generous contributions have supported this research.

\section{References}

1. Forastiere AA, Heitmiller RF, Kleinberg L. Multimodality therapy for esophageal cancer. Chest. 1997;112(4 Suppl):195S-200S.

2. Jackson C, Starling N, Chua YJ, Cunningham D. Pharmacotherapy for oesophagogastric cancer. Drugs. 2007;67:2539-56.

3. Pennathur A, Luketich JD. Resection for esophageal cancer: strategies for optimal management. Ann Thorac Surg. 2008;85:S751-6.

4. Reynolds JV, Muldoon C, Hollywood D, et al. Long-term outcomes following neoadjuvant chemoradiotherapy for esophageal cancer. Ann Surg. 2007;245:707-16.

5. Ancona E, Ruol A, Santi S, et al. Only pathologic complete response to neoadjuvant chemotherapy improves significantly the long term survival of patients with resectable esophageal squamous cell carcinoma: final report of a randomized, controlled trial of preoperative chemotherapy versus surgery alone. Cancer. 2001;91:2165-74.

6. Chirieac LR, Swisher SG, Ajani JA, et al. Posttherapy pathologic stage predicts survival in patients with esophageal carcinoma receiving preoperative chemoradiation. Cancer. 2005;103:1347-55.

7. Rohatgi P, Swisher SG, Correa AM, et al. Characterization of pathologic complete response after preoperative chemoradiotherapy in carcinoma of the esophagus and outcome after pathologic complete response. Cancer. 2005;104:2365-72.

8. Kleinberg L, Knisely JP, Heitmiller R, et al. Mature survival results with preoperative cisplatin, protracted infusion 5-fluorouracil, and 44-Gy radiotherapy for esophageal cancer. Int J Radiat Oncol Biol Phys. 2003;56:328-34.

9. Keats AS. The ASA classification of physical status - a recapitulation. Anesthesiology. 1978;49:233-6.
10. Heath EI, Burtness BA, Heitmiller RF, et al. Phase II evaluation of preoperative chemoradiation and postoperative adjuvant chemotherapy for squamous cell and adenocarcinoma of the esophagus. J Clin Oncol. 2000;18:868-76.

11. Greene FL, Fleming I, Fritz A, Balch C, Haller DG, eds. Esophagus. In: Cancer staging handbook. 6th ed. New York: Springer-Verlag; 2002.p.91-5.

12. Rohatgi PR, Swisher SG, Correa AM, et al. Failure patterns correlate with the proportion of residual carcinoma after preoperative chemoradiotherapy for carcinoma of the esophagus. Cancer. 2005;104:1349-55.

13. Urba SG, Orringer MB, Turrisi A, Iannettoni M, Forastiere A, Strawderman M. Randomized trial of preoperative cemoradiation versus surgery alone in patients with locoregional esophageal carcinoma. J Clin Oncol. 2001;19:305-13.

14. Kleinberg L, Brock MV, Jagannath SB, Forastiere A. Cancer of the esophagus. 4th ed. Philadelphia: Churchill Livingstone Elsevier; 2008.

15. Kelsen DP, Ginsberg R, Pajak TF, et al. Chemotherapy followed by surgery compared with surgery alone for localized esophageal cancer. N Engl J Med. 1998; 339:1979-84.

16. Takashima A, Shirao K, Hirashima Y, et al. Chemosensitivity of patients with recurrent esophageal cancer receiving perioperative chemotherapy. Dis Esophagus. 2008.

\section{Discussion}

Dr Michael Weyant (Aurora, Colo). Good morning. I would like to first thank the Western for inviting me to discuss this paper, and I would also like to thank the authors for sending me the manuscript well in advance.

In our evolution of the treatment of patients with locally advanced esophageal malignancy, trimodality therapy has emerged as the standard of care in this group of patients, with preoperative chemoradiation being the standard. What has emerged out of all this is the 3 groups of patients that you illustrated so nicely here, the patients with complete response responding better than either the patients with partial response or those with no response.

Your work illustrates the patterns of recurrences, which is also extremely valuable because it is work like this that will help guide future studies regarding the therapy of these patients as well.

I just have 3 brief questions for you.

First, I would like to know what your standard staging modalities are in the pretreatment setting for guiding your therapy of these patients.

Dr Meguid (Baltimore, $M d$ ). Although there has been an evolution of about 15 years between the first patients and our most recent patients, currently we are obtaining high-resolution CT scans of all patients. We are also increasingly using positron emission tomography scans and endoscopic ultrasound to stage patients.

Dr Weyant. My second question ties into that, knowing that you are using the modern staging modalities. In the manuscript you sent me, in the characterizations of your preclinically staged patients, you had 26 patients who were clinically staged as stage IV and conversely you had a group of patients of 12 , I believe, who were stage I that you did not illustrate in your talk. The questions are, what is the rationale and what are the characteristics of those patients in stage IV that actually led them to get surgery, and then conversely, what is the rationale for the group of patients in stage I to get the neoadjuvant therapy and then surgery as well?

Dr Meguid. I agree these are very valid points.

Those are all patients who got neoadjuvant therapy and surgery, and included in our series. At our institution, we offer neoadjuvant therapy and surgery to patients with Stage IVa disease.

These are limitations to our preliminary study, and we need to resolve these before we pursue this as a publication. 
Dr Weyant. The last question is, and you briefly alluded to the difference between squamous carcinomas and adenocarcinomas. There is a general belief that they behave very differently and respond differently to neoadjuvant therapy. Did you do any subset analysis looking at the 2 subgroups regarding complete response and survival?

Dr Meguid. Yes, actually this is in the manuscript revised since we sent it to you. In brief, the patients with nonresponding squamous cell carcinoma did very poorly and died very rapidly, whereas the patients with squamous cell carcinoma whom responded had relatively favorable outcomes, and have not yet reached $50 \%$ mortality. The differences in patients with adenocarcinoma are less pronounced than this. Again, we have done a series of subanalyses and are currently trying to identify which different relationships exist.

Dr David Follette (Sacramento, Calif). That was a superbly presented paper. I am always pleased to see when studies validate other work. The subset of your squamous cell carcinoma certainly validates some work from East Germany that showed in a group of patients showing complete response with squamous cell carcinomas there was approximately 70\% 5-year survival. I think your validation of that work is very important and in a way validates some of your conclusions, notwithstanding the homework you are going to do when you get back.

Having said that, it is like what we were talking about yesterday with lung cancer. At Baltimore, do you do any restaging after neoadjuvant chemoradiation?

Dr Meguid. We do some restaging, mostly the $50 \%$ of our patients who get their neoadjuvant therapy at outside institutions. In this study, we did not look at that restaging. We looked at patients' preneoadjuvant staging and their postoperative pathologic staging to make a comparison as to who was downstaged, who showed no response, and who had a complete response.

Dr Follette. Because as you know, we have no prospective randomized trials validating neoadjuvant treatment, but if we begin to look-and Tom Rice has pointed this out to us on numerous occasions. Nodal disease is really a poor indicator.

My second question, when you are talking about complete response, you are talking about both the tumor and no positive nodes in the surgical specimen?

Dr Meguid. That is correct. There is no evidence of disease in the surgical specimen.

Dr Follette. I appreciate a very well-presented paper, and I believe your results are going to help all of us in the debate about neoadjuvant treatment and its value in that subset of patients who show complete response.

Dr Robert Cerfolio (Birmingham, Ala). Very well presented. Two quick questions, because I see the time is short. Do you have maximum standardized uptake value data in the people who got the positron emission tomography, the change in the maximum standardized uptake value?

Dr Meguid. We do not have that data in our database.

Dr Cerfolio. So can I look forward then to seeing it in the manuscript?

Dr Meguid. No, it won't be.

Dr Cerfolio. Okay, the second question is: I love to come to these meetings because I learn stuff and I take it back. You heard how I changed my practice with Dr Lowe's article a few years ago. How is this paper going to change my practice? What am I going to do differently?

Dr Meguid. This may change what you tell your patients. If you have female patients, if you have patients with squamous cell esophageal adenocarcinoma, you may be able to tell them they are more likely to show complete response than male patients or patients with adenocarcinoma. That is in the short run. In the long run, I believe this is leading us more to look at tumor biology differences and try to identify what we can do to appropriately target patients with better chemotherapeutic regimens so that we can ultimately have more clinical complete responses as opposed to just continuing the same trends we have been doing.

Dr James Stewart (Colorado Springs, Colo). As a 20-year veteran of advocating neoadjuvant chemoradiation for esophageal cancer, we noticed about 15 years ago that patients showing partial response just did not do as well, and although I have not published any information, we developed some postoperative treatment strategies for those patients and wondered if you had also adopted something similar.

Dr Meguid. That is an interesting observation. In fact, patients showing partial response do almost as poorly as those showing no response, such that in the abstract in the meeting program, we combined them because they have similarly dismal outcomes. Our patients-about $20 \%$ of all the groups, with no statistically significant difference between the groups-received postoperative chemotherapy. I do not believe it resulted in a difference in the 3 treatment groups. As of yet, we have not been able to identify, up front, patients whom are going to have partial response versus those going to have no response, unfortunately. 